\title{
Potential role of myeloid cell/eosinophil-derived IL-17 in LPS-induced endotoxin shock
}

\section{Short title: IL-17 cytokines in LPS-induced endotoxin shock}

Eri Shimura ${ }^{1}$, Akiko Shibui ${ }^{2}$, Seiko Narushima ${ }^{3}$, Aya Nambu ${ }^{3}$, Sachiko Yamaguchi $^{3}$, Aoi Akitsu $^{4}$, Warren J. Leonard ${ }^{5}$, Yoichiro Iwakura ${ }^{4}$, Kenji Matsumoto, ${ }^{6}$ Hajime Suto ${ }^{1}$, Ko Okumura ${ }^{1}$, Katsuko Sudo ${ }^{7}$ and Susumu Nakae $e^{3,8 *}$

1 Atopy Research Center, Juntendo University, Tokyo 113-8412, Japan

2 Department of Medical Genomics, Graduate School of Frontier Sciences, The University of Tokyo, Chiba 277-8561, Japan

3 Laboratory of Systems Biology, Center for Experimental Medicine and Systems Biology, The Institute of Medical Science, The University of Tokyo, Tokyo 108-8639, Japan

4 Division of Experimental Animal Immunology, Tokyo University of Science, Chiba 278-8510, Japan

5 Laboratory of Molecular Immunology, National Heart, Lung, and Blood Institute, National Institutes of Health, Bethesda, MD 20892, USA

6 Department of Allergy and Immunology, National Research Institute for Child Health and Development, Tokyo 157-8535, Japan

7 Animal Research Center, Tokyo Medical University, Tokyo 160-8402, Japan 
8 Precursory Research for Embryonic Science and Technology (PRESTO), Japan Science and Technology Agency, Saitama 332-0012, Japan

* Address correspondence to:

Susumu Nakae, Ph.D.

Laboratory of Systems Biology, Center for Experimental Medicine and Systems

Biology, The Institute of Medical Science

The University of Tokyo

4-6-1 Shirokanedai, Minato, Tokyo, 108-8639, Japan

Tel: +81-3-6409-2111, Fax: +81-3-6409-2109

E-mail: snakae@ims.u-tokyo.ac.jp

KEY WORDS: IL-17A, IL-17F, IL-25, sepsis 


\section{Abstract}

IL-17RA is a shared receptor subunit for several cytokines of the IL-17 family, including IL-17A, IL-17C, IL-17E (also called IL-25) and IL-17F. It has been shown that mice deficient in IL-17RA are more susceptible to sepsis than wild-type mice, suggesting that IL-17RA is important for host defense against sepsis. However, it is unclear which ligands for IL-17RA, such as IL-17A, IL-17C, IL-17E/IL-25 and/or IL-17F, are involved in the pathogenesis of sepsis. Therefore, we examined IL-17A, IL-17E/IL-25 and IL-17F for possible involvement in LPS-induced endotoxin shock. IL-17A-deficient mice, but not IL-25- or IL-17F-deficient mice, were highly susceptible to LPS-induced endotoxin shock compared with wild-type mice. On the other hand, using IL-6-deficient, IL-21R $\alpha$-deficient and Rag-2-deficient mice, IL-6 and IL-21, cytokines that are important for Th17 cell differentiation, and Th17 cells were not essential for development of LPS-induced endotoxin shock, suggesting that certain IL-17A-producing cells other than Th17 cells were important in the setting. In this connection, IL-17A was produced by macrophages, DCs and eosinophils after LPS injection. Taken together, these findings indicate that IL-17A, but not IL-17F or IL-25, is crucial for LPS-induced endotoxin shock. In addition, macrophages, DCs and eosinophils, but not Th17 cells or $\gamma \delta$ T cells, may be sources of IL-17A during LPS-induced endotoxin shock. 


\section{Introduction}

To date, at least six genes, i.e., interleukin-17 (IL-17, also called IL-17A), IL-17B, IL-17C, IL-17D, IL-17E (also called IL-25) and IL-17F, and five genes, i.e., IL-17RA, IL-17RB, IL-17RC, IL-17RD and IL-17RE, have been identified as members of the IL-17 cytokine and IL-17R receptor families, respectively [1,2]. IL-17A and IL-17F bind to IL-17R (composed of IL-17RA and IL-17RC), IL-17C binds to IL-17CR (composed of IL-17RA and IL-17RE) and IL-25 binds to IL-25R (composed of IL-17RA and IL-17RB) [3]. However, the receptors for IL-17B and IL-17D have not been fully elucidated, although IL-17B is known to bind to IL-17RB [4]. Thus, excluding IL-17B and IL-17D, IL-17RA is considered to be a shared receptor subunit for many IL-17 cytokines, including IL-17A, IL-17C, IL-17F and IL-25.

Administration and/or overexpression of IL-17A, IL-17B, IL-17C, IL-17D and/or IL-17F in mice resulted in development of neutrophilic inflammation by inducing neutrophil chemoattractants [5,6,7], and this contributed to host defense against various pathogens [8]. On the other hand, administration and/or overexpression of IL-25 in mice resulted in development of eosinophilic inflammation by inducing Th2 cytokines such as IL-4, IL-5 and IL-13 $[9,10,11]$, which contributed to host defense against nematodes $[12,13,14]$. However, inappropriate and excessive production of these cytokines is also involved in development of chronic inflammatory diseases such as autoimmune and allergic diseases $[1,15]$. In particular, inappropriate and/or excessive IL-17A or IL-17F, which are produced by various types of cells, such as Th17 cells, $\gamma \delta$ T cells, iNKT cells, NK cells and/or LTi cells [16,17], contributes to development of rheumatoid arthritis, 
multiple sclerosis, inflammatory bowel diseases, psoriasis, asthma and/or contact dermatitis [1]. IL-17A and IL-17F may also be involved in induction of acute inflammation such as sepsis. Indeed, the IL-17A and IL-17F levels were increased in mice with sepsis induced by caecal ligation and puncture (CLP) $[18,19,20]$. In addition, mice treated with neutralizing Ab for IL-17A were resistant to sepsis induced by CLP, suggesting that IL-17A, especially $\gamma \delta \mathrm{T}$ cell-derived IL-17A, promotes sepsis [18]. In contrast, IL-17A-deficient (IL-17A ${ }^{-/}$) mice were more susceptible to sepsis induced by CLP than wild-type mice, suggesting that IL-17A helps protect against sepsis [21]. Therefore, the precise role of IL-17A in the development of sepsis following CLP remains controversial. In addition, like the $\mathrm{IL}_{-17 \mathrm{~A}^{-/}}$mice [21], IL-17RA ${ }^{-/}$mice were also more susceptible to CLP-induced sepsis than wild-type mice [22]. Since, as noted above, IL-17RA is a shared receptor subunit for IL-17A, IL-17C, IL-17F and IL-25, it is unclear if the

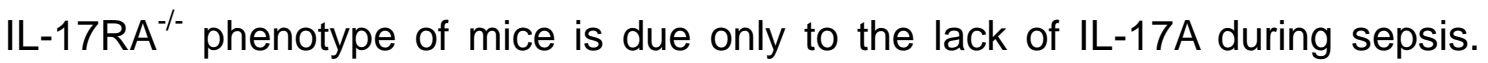

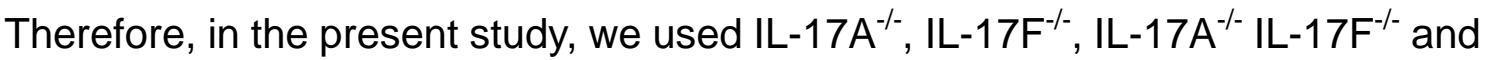
IL-25/- mice to investigate the roles of IL-17A, IL-17F and IL-25 in sepsis induced by LPS injection. We found that IL-17A, but not IL-17F or IL-25, produced by macrophages was crucial for LPS-induced endotoxin shock. 


\section{Materials and Methods}

\subsection{Mice}

C57BL/6J wild-type mice were purchased from SLC Japan. IL-17A ${ }^{-/-}$, IL-17F ${ }^{-/}$, and $\mathrm{IL}-17 \mathrm{~A}^{-/-} \mathrm{IL}-17 \mathrm{~F}^{-/-}$mice on the $\mathrm{C} 57 \mathrm{BL} / 6 \mathrm{~J}$ background were generated as described elsewhere $[23,24]$. IL-25 $5^{+/-}$mice were obtained by mating male chimeric mice-which were generated by Lexicon Pharmaceuticals, Inc. using il25-targeted 129 ES cells (OYC069)—with C57BL/6J female mice (N8) [25]. IL-17A-green fluorescent protein (GFP) reporter mice were generated by Y.I. (unpublished). C57BL/6-Rag-2 ${ }^{-/-}$mice and C57BL/6-IL-6 ${ }^{-/-}$mice were obtained from Taconic Farm and Jackson Laboratories, respectively. C57BL/6J-IL-21R $\alpha^{-/-}$ mice were generated as described elsewhere [26]. Eight- to 12-wk-old male mice were used in all experiments. All mice were housed under specific pathogen-free conditions in an environmentally-controlled clean room at The Institute of Medical Science, The University of Tokyo. All animal experiments were approved by the Institutional Review Board of The Institute of Medical Science, The University of Tokyo, and conducted in accordance with the ethical and safety guidelines of the institution (A20-12).

\subsection{LPS-induced endotoxin shock}

Mice were intraperitoneally injected with $10-15 \mathrm{mg} / \mathrm{kg}$ of LPS (Escherichia coli serotype 0111:B4; Sigma-Aldrich). After LPS injection, the survival of the mice was monitored for 5 days.

\subsection{Preparation of peritoneal cells}


Six hours after LPS injection, mice were intraperitoneally injected with $2 \mathrm{ml}$ of PBS. Peritoneal cells were then collected in the peritoneal lavage fluids from the mice.

\subsection{Measurement of cytokines}

The concentrations of IL-6, IL-17A, IL-17F, IL-21, IL-25, IL-6 and TNF in the peritoneal lavage fluids from LPS-injected mice were measured using ELISA kits (eBioscience or BioLegend), according to the manufacturer's instructions.

\subsection{Flow cytometry analysis}

Peritoneal cells were incubated with anti-CD16/CD32 mAb (93, eBioscience) in FACS buffer (phosphate-buffered saline containing $1 \%$ inactivated fetal calf serum and $0.1 \% \mathrm{NaN}_{3}$ ) for 15 min on ice and then incubated with PE-conjugated anti-mouse CD45R/B220 (RA3-6B2; BD Bioscience), PE/Cy7-conjugated anti-mouse CD11b (M1/70; BD Bioscience), BD Horizon V500 anti-mouse CD3ع (500A2; BD Bioscience), APC-conjugated anti-mouse F4/80 (BM8; BioLegend) and APC/Cy7-conjugated anti-mouse CD11c (N418; BD BioLegend) mAbs for 25 min on ice. After washing, the cells were suspended in FACS buffer containing 7-amino actinomycin D, and determined with a MACSQuant (Miltenyi Biotec), and analyzed with FlowJo software (Tree Star).

\subsection{Statistical analysis}

The Kaplan-Meier method using the log-rank test was used for statistical evaluation of animal survival. Unless otherwise specified, the unpaired Student's 
$t$-test, two-tailed, was used for statistical evaluation of the results. 


\section{Results}

\subsection{IL-17A, but not IL-17F or IL-25, is important for LPS-induced endotoxin}

shock.

Proinflammatory cytokines such as TNF are known to be important for the pathogenesis of LPS-induced endotoxin shock [27]. Indeed, the levels of TNF as well as IL-6 increased rapidly and significantly in the peritoneal fluids of C57BL/6 wild-type mice after LPS injection (Fig. 1A). The levels of IL-17A, but not IL-17F, IL-25 or IL-21, were also increased in the setting (Fig. 1A), suggesting that IL-17A may be involved in LPS-induced endotoxin shock.

To clarify this, we injected LPS intraperitoneally to mice deficient in IL-17A, $\mathrm{IL}-17 \mathrm{~F}$ or $\mathrm{IL}-25$. As shown in Figure $1 \mathrm{~B}, \mathrm{IL}-17 \mathrm{~A}^{-/-}$mice as well as $\mathrm{IL}_{-17 \mathrm{~A}^{-/}}$ IL-17F ${ }^{-/-}$mice were resistant to LPS-induced endotoxin shock compared with wild-type mice. On the other hand, IL-17F ${ }^{-/-}$mice and $\mathrm{IL}-25^{-/-}$mice showed susceptibility similar to that of wild-type mice for LPS-induced endotoxin shock (Fig. 1B). These observations indicate that IL-17A, but not IL-17F or IL-25, is crucial for induction of endotoxin shock by LPS.

\subsection{Myeloid cells and eosinohils, but not Th17 cells, is a source of IL-17A} during LPS-induced endotoxin shock.

To identify the types of cells producing IL-17A during LPS-induced endotoxin shock, we injected LPS intraperitoneally to IL-17A reporter mice, which express EGFP simultaneously with IL-17A. Six hours later, EGFP-positive CD45 ${ }^{+}$cells were observed in the peritoneal fluids (Fig. 2). Furthermore, the CD45 ${ }^{+}$EGFP $^{+}$ cells were identified as $\mathrm{TCR}^{+}$and $\mathrm{TCR} \gamma^{+} \mathrm{CD} 3 \varepsilon^{+} \mathrm{CD} 4^{+} \mathrm{T}$ cells, but not $\mathrm{CD} 3^{+}$ 
$\mathrm{CD}^{+} \mathrm{T}$ cells or TCR $\beta^{+} \mathrm{CD}^{+} \mathrm{DX} 5 / \mathrm{CD} 49 \mathrm{~d}^{+}$cells (Fig. $3 \mathrm{~A}$ ), suggesting that Th17 cells and $\gamma \delta \mathrm{T}$ cells, but not CD8 ${ }^{+} \mathrm{T}$ cells or NKT cells, are potential sources of IL-17A in the setting. In addition, Siglec $\mathrm{F}^{+} \mathrm{CD} 11 \mathrm{~b}^{+}$cells (eosinophils), MHC class II ${ }^{\text {hilint }} \mathrm{F} 4 / 80^{\text {hilint }}$ cells (monocytes/macrophages), MHC class $\mathrm{II}^{+} \mathrm{CD} 11 \mathrm{c}^{+}$ cells (DCs) and $\mathrm{B} 220^{+} \mathrm{CD} 19^{+}$cells (B cells), but not $\mathrm{CD}_{11 \mathrm{~b}^{+} \mathrm{Gr}^{+}}$cells (neutrophils) were identified as producers of IL-17A (Fig. 3B). Therefore, in addition to Th17 cells and $\gamma \delta$ T cells, eosinophils, macrophages, DCs and B cells are also potential sources of IL-17A during LPS-induced endotoxin shock.

Th17 cells, which differentiate from naïve $\mathrm{CD}^{+} \mathrm{T}$ cells in the presence of TGF- $\beta$, IL-6 and/or IL-21 [28], are known to be a major source of IL-17A and IL-17F [28], Consistent with a previous report [29], IL-6 $6^{-/-}$mice showed similar susceptibility to LPS-induced endotoxin shock as wild-type mice (Fig. 4A). Likewise, the susceptibility to LPS-induced endotoxin shock was comparable among wild-type, IL-21R $\alpha^{-/-}$and IL-6 ${ }^{-/-} \mathrm{IL}-21 \mathrm{R} \alpha^{-/-}$mice (Fig. 4A). These observations suggest that IL-6 and IL-21R are not essential for IL-17A-mediated LPS-induced endotoxin shock. In addition, in contrast to IL-17A ${ }^{-/-}$mice (Fig. 1B), Rag- $2^{-/-}$mice, which lack T cells, B cells and NKT cells, were more highly susceptible to LPS-induced endotoxic shock than wild-type mice were (Fig. 4B), suggesting that such cells are important for suppression of LPS-induced endotoxin shock. Taken together, these findings suggest that IL-17A derived from macrophages, DCs and/or eosinophils, but not T cells, B cells or NKT cells, is crucial for induction of LPS-induced endotoxin shock. 


\section{Discussion}

$\mathrm{IL}^{-17 \mathrm{RA}^{-/-}}$mice were reported to be more susceptible to sepsis induced by CLP compared with wild-type mice [22], suggesting involvement of ligands for IL-17RA, such as IL-17A, IL-17C, IL-17F and IL-25, in induction of sepsis. On the other hand, the role of IL-17A to induction of sepsis by CLP has been controversial. That is, mice treated with neutralizing Ab for IL-17A were resistant [18] , but IL-17A ${ }^{-/-}$mice were susceptible [21], to sepsis induced by CLP. Therefore, it is unclear which ligand(s) for IL-17RA is crucial for the response. In

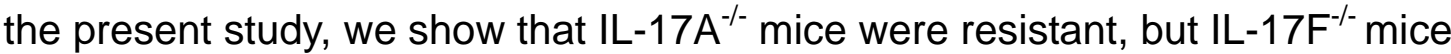
and IL-25 $5^{-/}$mice were normally susceptible, to LPS-induced endotoxin shock. That suggests that IL-17A, but not IL-17F or IL-25, is an important effector cytokine for LPS-induced endotoxin shock, although it should be noted that the pathogenic mechanism of the LPS-induced and CLP-induced sepsis models is not exactly the same [30]. We also found that macrophages, DCs and eosinophils-but not T cells (such as Th17 cells, CD8 ${ }^{+} T$ cells and $\gamma \delta$ T cells), B cells or NKT cells-were potential sources of IL-17A in LPS-induced endotoxin shock. Although it was reported that $\gamma \delta$ T-cell-derived IL-17A may contribute to aggravation of CLP-induced sepsis [18], the contributions of T cells and B cells to induction of sepsis are controversial. In this regard, while it was reported that T cells, especially regulatory $T$ cells (Treg cells), and B cells were important for protection against CLP-induced or microbe-induced sepsis [31,32], others reported that these cells were not involved in CLP-induced sepsis $[33,34]$. We showed that IL-17A was rapidly expressed in Th17 cells, $\gamma \delta$ T cells, B cells, 
macrophages and eosinophils, but not in $\mathrm{CD}^{+} \mathrm{T}$ cells, NKT cells or neutrophils, in IL-17A reporter mice after intraperitoneal LPS injection. However, IL-6 and IL-21, which are important for the development of Th17 cells, were not essential for LPS-induced endotoxin shock. In addition, Rag-2 ${ }^{-/-}$mice, which lack T cells, B cells and NKT cells, were susceptible to LPS-induced endotoxin shock, while $\mathrm{IL}-17 \mathrm{~A}^{-/-}$mice were resistant to it. These observations suggest that $\mathrm{T}$ cells, $\mathrm{B}$ cells and/or NKT cells play protective roles against LPS-induced endotoxin shock. Therefore, Th17 cell- and $\gamma \delta$ T cell-derived IL-17 was not essential for development of LPS-induced endotoxin shock.

In summary, IL-17A, but not IL-17F or IL-25, is crucial for LPS-induced endotoxin shock. In addition, macrophages, DCs and eosinophils, but not Th17 cells or $\gamma \delta$ T cells, may be sources of that IL-17A during LPS-induced endotoxin shock.

\section{Acknowledgements}

We thank Wakako Nakanishi, Yoshihisa Hiraishi, Ayano Yamauchi-Ishii, Tsubasa Sato and Hiromi Iwami for their technical assistance. We also thank Lawrence W. Stiver (Tokyo, Japan) for his critical reading of the manuscript. 


\section{References}

1. Iwakura $Y$, Ishigame $H$, Saijo $S$, Nakae $S$ (2011) Functional specialization of interleukin-17 family members. Immunity 34: 149-162.

2. Miossec P, Kolls JK (2012) Targeting IL-17 and TH17 cells in chronic inflammation. Nat Rev Drug Discov 11: 763-776.

3. Rickel EA, Siegel LA, Yoon BR, Rottman JB, Kugler DG, et al. (2008) Identification of functional roles for both IL-17RB and IL-17RA in mediating IL-25-induced activities. J Immunol 181: 4299-4310.

4. Gaffen SL (2009) Structure and signalling in the IL-17 receptor family. Nat Rev Immunol 9: 556-567.

5. Li H, Chen J, Huang A, Stinson J, Heldens S, et al. (2000) Cloning and characterization of IL-17B and IL-17C, two new members of the IL-17 cytokine family. Proc Natl Acad Sci U S A 97: 773-778.

6. Shi Y, Ullrich SJ, Zhang J, Connolly K, Grzegorzewski KJ, et al. (2000) A novel cytokine receptor-ligand pair. Identification, molecular characterization, and in vivo immunomodulatory activity. J Biol Chem 275: 19167-19176.

7. Yang XO, Chang SH, Park H, Nurieva R, Shah B, et al. (2008) Regulation of inflammatory responses by IL-17F. J Exp Med 205: 1063-1075.

8. Iwakura Y, Nakae S, Saijo S, Ishigame H (2008) The roles of IL-17A in inflammatory immune responses and host defense against pathogens. Immunol Rev 226: 57-79.

9. Kim MR, Manoukian R, Yeh R, Silbiger SM, Danilenko DM, et al. (2002) Transgenic overexpression of human IL-17E results in eosinophilia, B-lymphocyte hyperplasia, and altered antibody production. Blood 100: 2330-2340.

10. Price AE, Liang HE, Sullivan BM, Reinhardt RL, Eisley CJ, et al. (2010) Systemically dispersed innate IL-13-expressing cells in type 2 immunity. Proc Natl Acad Sci U S A 107: 11489-11494.

11. Saenz SA, Siracusa MC, Perrigoue JG, Spencer SP, Urban JF, Jr., et al. (2010) IL25 elicits a multipotent progenitor cell population that promotes $\mathrm{T}(\mathrm{H}) 2$ cytokine responses. Nature 464: 1362-1366.

12. Owyang AM, Zaph C, Wilson EH, Guild KJ, McClanahan T, et al. (2006) Interleukin 25 regulates type 2 cytokine-dependent immunity and limits chronic inflammation in the gastrointestinal tract. J Exp Med 203: 843-849.

13. Fallon PG, Ballantyne SJ, Mangan NE, Barlow JL, Dasvarma A, et al. (2006) Identification of an interleukin (IL)-25-dependent cell population that provides IL-4, IL-5, and IL-13 at the onset of helminth expulsion. J Exp Med 203: 1105-1116.

14. Zhao A, Urban JF, Jr., Sun R, Stiltz J, Morimoto M, et al. (2010) Critical role of IL-25 in nematode infection-induced alterations in intestinal function. $J$ Immunol 185: 6921-6929.

15. Oboki K, Ohno T, Saito H, Nakae S (2008) Th17 and allergy. Allergol Int 57: 121-134. 
16. Korn T, Bettelli E, Oukka M, Kuchroo VK (2009) IL-17 and Th17 Cells. Annu Rev Immunol 27: 485-517.

17. Cua DJ, Tato CM (2010) Innate IL-17-producing cells: the sentinels of the immune system. Nat Rev Immunol 10: 479-489.

18. Flierl MA, Rittirsch D, Gao H, Hoesel LM, Nadeau BA, et al. (2008) Adverse functions of IL-17A in experimental sepsis. FASEB J 22: 2198-2205.

19. Bosmann M, Patel VR, Russkamp NF, Pache F, Zetoune FS, et al. (2011) MyD88-dependent production of IL-17F is modulated by the anaphylatoxin $\mathrm{C5a}$ via the Akt signaling pathway. FASEB $\mathrm{J} 25$ : 4222-4232.

20. Bosmann M, Meta F, Ruemmler R, Haggadone MD, Sarma JV, et al. (2013) Regulation of IL-17 family members by adrenal hormones during experimental sepsis in mice. Am J Pathol 182: 1124-1130.

21. Ogiku M, Kono H, Hara M, Tsuchiya M, Fujii H (2012) Interleukin-17A plays a pivotal role in polymicrobial sepsis according to studies using IL-17A knockout mice. J Surg Res 174: 142-149.

22. Freitas A, Alves-Filho JC, Victoni T, Secher T, Lemos HP, et al. (2009) IL-17 receptor signaling is required to control polymicrobial sepsis. $\mathrm{J}$ Immunol 182: 7846-7854.

23. Nakae S, Komiyama Y, Nambu A, Sudo K, Iwase M, et al. (2002) Antigen-specific $T$ cell sensitization is impaired in IL-17-deficient mice, causing suppression of allergic cellular and humoral responses. Immunity 17: 375-387.

24. Ishigame H, Kakuta S, Nagai T, Kadoki M, Nambu A, et al. (2009) Differential roles of interleukin-17A and -17F in host defense against mucoepithelial bacterial infection and allergic responses. Immunity 30: 108-119.

25. Ishii A, Oboki K, Nambu A, Morita H, Ohno T, et al. (2010) Development of IL-17-mediated delayed-type hypersensitivity is not affected by down-regulation of IL-25 expression. Allergol Int 59: 399-408.

26. Ozaki K, Spolski R, Feng CG, Qi CF, Cheng J, et al. (2002) A critical role for IL-21 in regulating immunoglobulin production. Science 298: 1630-1634.

27. Pasparakis M, Alexopoulou L, Episkopou V, Kollias G (1996) Immune and inflammatory responses in TNF alpha-deficient mice: a critical requirement for TNF alpha in the formation of primary $B$ cell follicles, follicular dendritic cell networks and germinal centers, and in the maturation of the humoral immune response. J Exp Med 184: 1397-1411.

28. Dong C (2008) TH17 cells in development: an updated view of their molecular identity and genetic programming. Nat Rev Immunol 8: 337-348.

29. Dalrymple SA, Slattery R, Aud DM, Krishna M, Lucian LA, et al. (1996) Interleukin- 6 is required for a protective immune response to systemic Escherichia coli infection. Infect Immun 64: 3231-3235.

30. Remick DG, Newcomb DE, Bolgos GL, Call DR (2000) Comparison of the mortality and inflammatory response of two models of sepsis: lipopolysaccharide vs. cecal ligation and puncture. Shock 13: 110-116.

31. Heuer JG, Zhang T, Zhao J, Ding C, Cramer M, et al. (2005) Adoptive transfer of in vitro-stimulated CD4+CD25+ regulatory $T$ cells increases 
bacterial clearance and improves survival in polymicrobial sepsis. J Immunol 174: 7141-7146.

32. Rauch PJ, Chudnovskiy A, Robbins CS, Weber GF, Etzrodt M, et al. (2012) Innate response activator B cells protect against microbial sepsis. Science 335: 597-601.

33. Bosmann M, Russkamp NF, Patel VR, Zetoune FS, Sarma JV, et al. (2011) The outcome of polymicrobial sepsis is independent of $\mathrm{T}$ and $\mathrm{B}$ cells. Shock 36: 396-401.

34. Scumpia PO, Delano MJ, Kelly KM, O'Malley KA, Efron PA, et al. (2006) Increased natural CD4+CD25+ regulatory $T$ cells and their suppressor activity do not contribute to mortality in murine polymicrobial sepsis. J Immunol 177: 7943-7949. 


\section{Figure legends}

Figure 1. IL-17A, but not IL-17F or IL-25, is important for LPS-induced endotoxin shock.

(A) C57BL/6J-wild-type mice were intraperitoneally injected with LPS (15 mg/kg), and peritoneal lavage fluids were collected. The concentrations of IL-17A, IL-17E, IL-17F, IL-6, IL-21 and TNF in the fluids were measured by ELISA. Data show the mean \pm SEM. $N=10(0 \mathrm{~h}), 4(1 \mathrm{~h}), 9(3 \mathrm{~h})$ and $10(6 \mathrm{~h}) .{ }^{*} \mathrm{p}<0.05$ and ${ }^{* *} \mathrm{p}<0.005$ vs. 0 h. (B) Wild-type mice $(n=27), I L-17 A^{-/-}$mice $(n=14), I L-17 F^{-/-}$

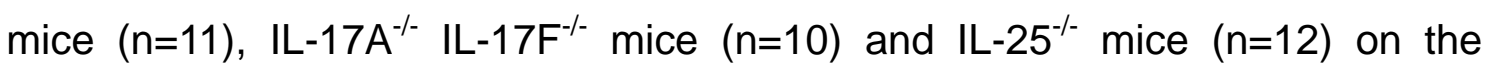
C57BL/6J background were intraperitoneally injected with LPS $(10 \mathrm{mg} / \mathrm{kg})$. The viability of these mice was monitored daily. Data show pooled data from 2 independent experiments. ${ }^{*} p<0.001$ and ${ }^{* *} p<0.005$ vs. wild-type mice.

Figure 2. Detection of IL-17A-producing cells in peritoneal lavage fluids of mice after intraperitoneal LPS injection.

IL-17A-EGFP reporter mice were intraperitoneally injected with $15 \mathrm{mg} / \mathrm{kg}$ LPS, and peritoneal lavage fluids were collected $6 \mathrm{~h}$ later. IL-17A-producing EGFP ${ }^{+}$ cells in 7-aminoactinomycin D-negative $\mathrm{CD} 45^{+}$peritoneal cells were detected by flow cytometry. Data show a representative result from 4 independent experiments.

Figure 3. Identification of IL-17A-producing cells in peritoneal lavage fluids of mice after intraperitoneal LPS injection.

Cell lineage markers on the 7-aminoactinomycin D-negative $\mathrm{CD}_{4} 5^{+} \mathrm{EGFP}^{+}$ 
peritoneal cells obtained from the LPS-injected IL-17A-EGFP reporter mice described in Figure 3 were determined by flow cytometry. Data show a representative result from 3 independent experiments.

Figure 4. IL-6 and IL-21R $\alpha$ are dispensable for LPS-induced endotoxin shock.

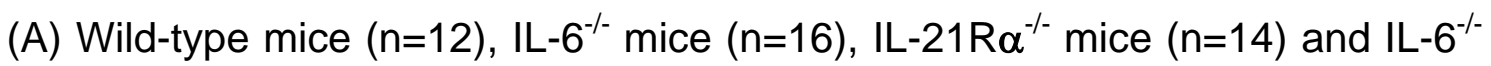
IL-21R $\alpha^{-/-}$mice $(n=15)$ on the C57BL/6J background were intraperitoneally injected with LPS (15 mg/kg). (B) Wild-type mice $(n=50)$, and Rag- $2^{-/}$mice $(n=52)$ on the C57BL/6J background were intraperitoneally injected with LPS (15 $\mathrm{mg} / \mathrm{kg}$ ). The viability of these mice was monitored daily. Data show pooled data from 2 independent experiments. ${ }^{*} p<0.05$ vs. wild-type mice. 
Figure 1. Shimura et al.

A
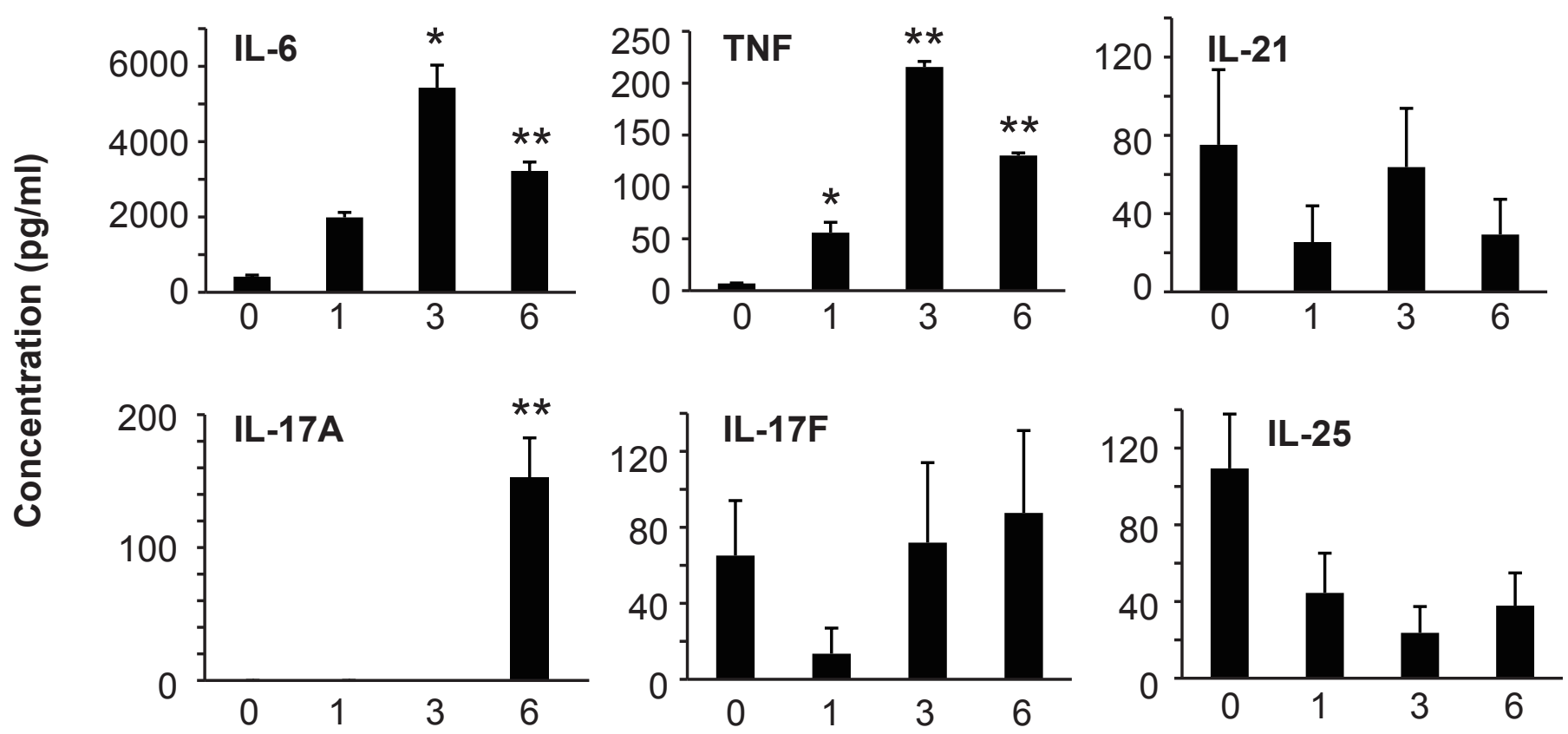

Time after LPS injection (h)

B

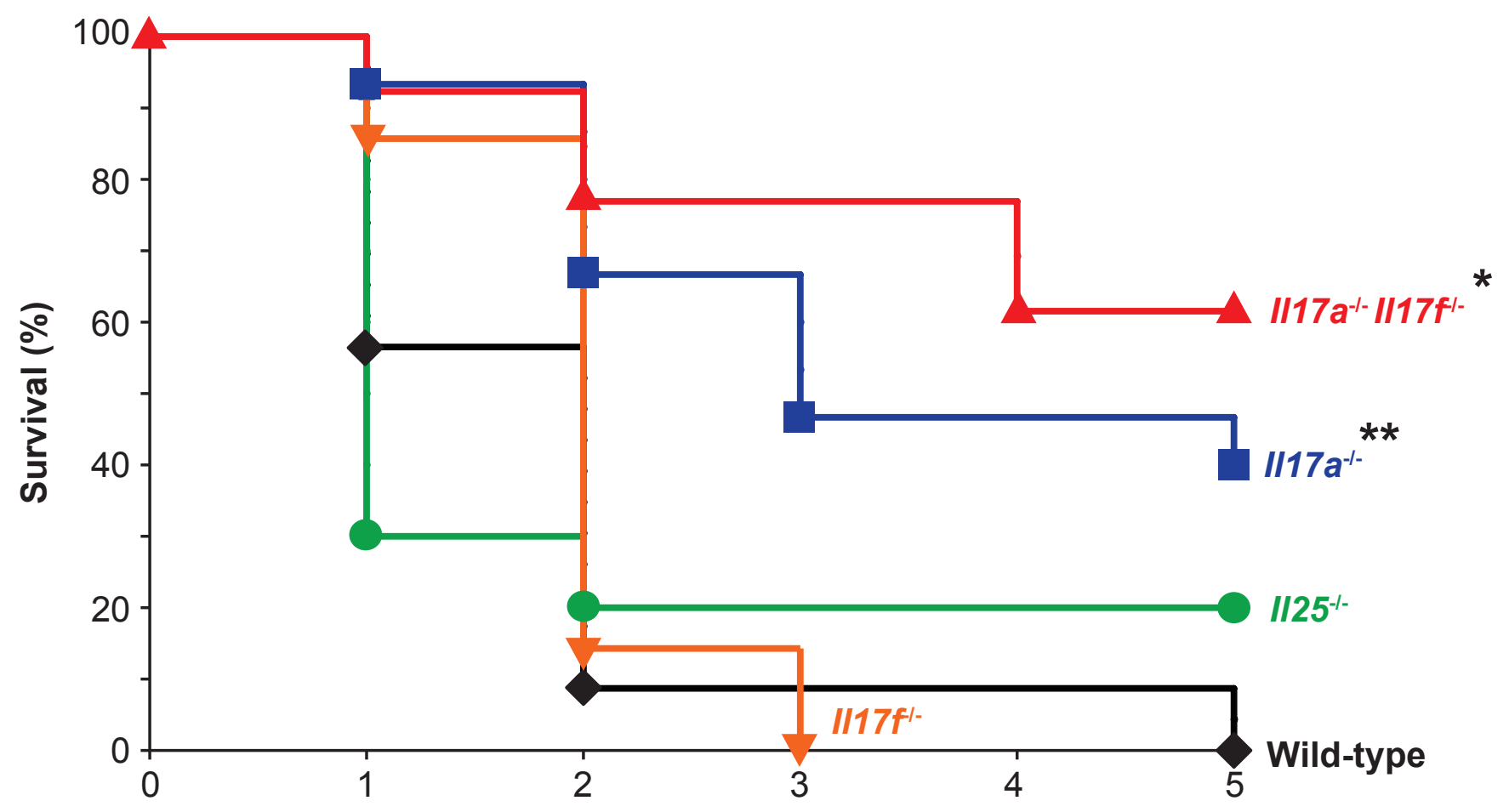

Time after LPS injection (days) 
Figure 2

Figure 2. Shimura et al.

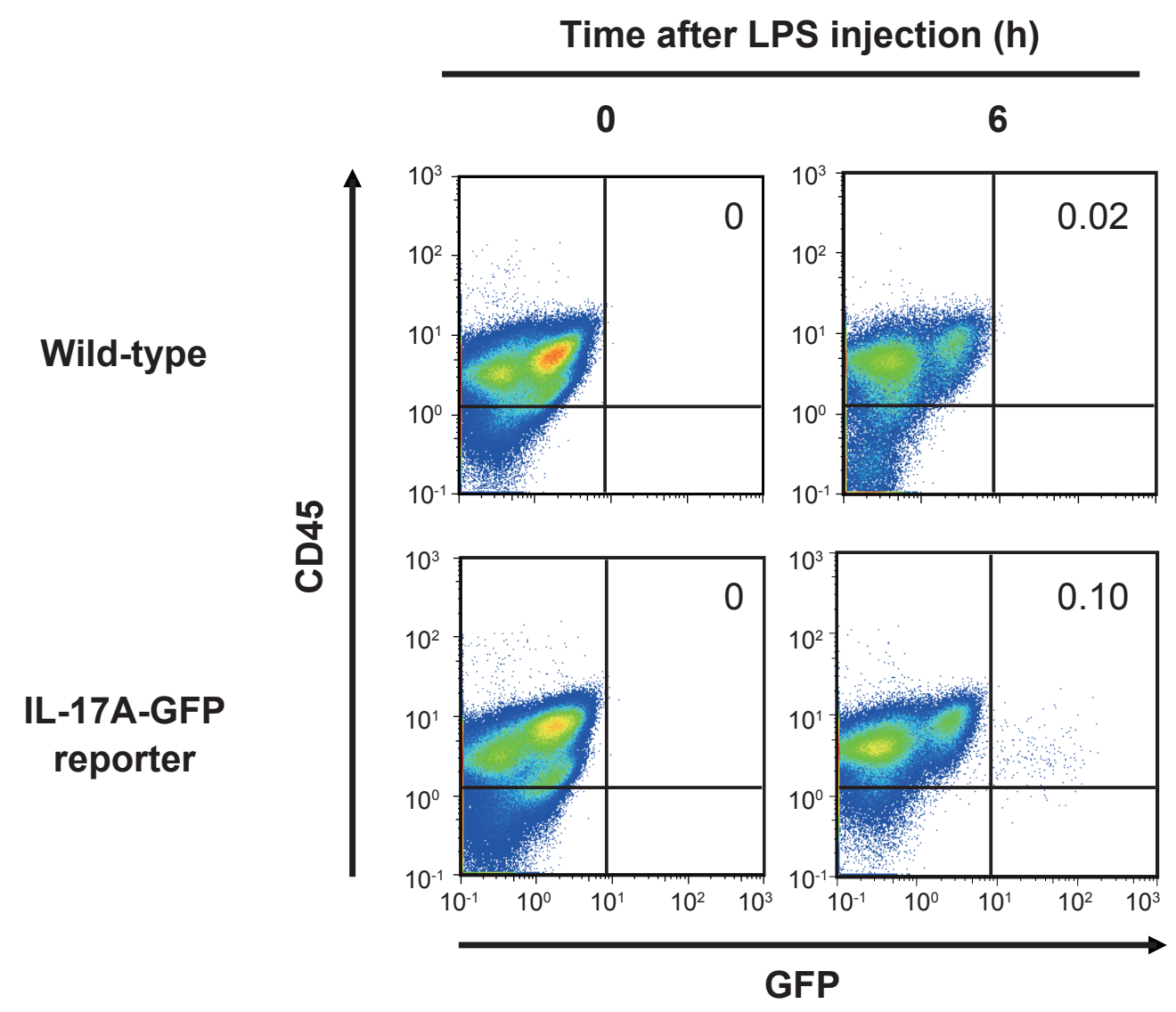


Figure 3. Shimura et al.

A
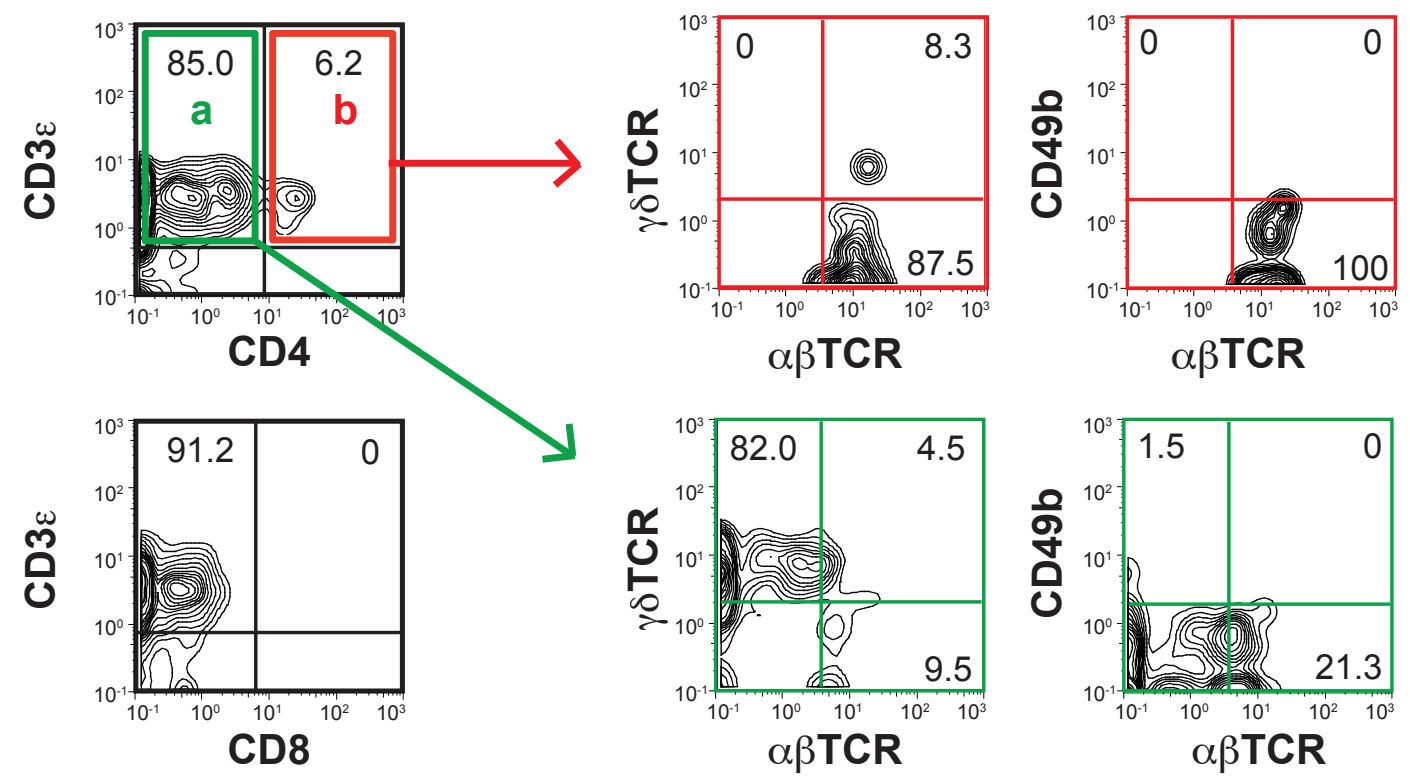

B
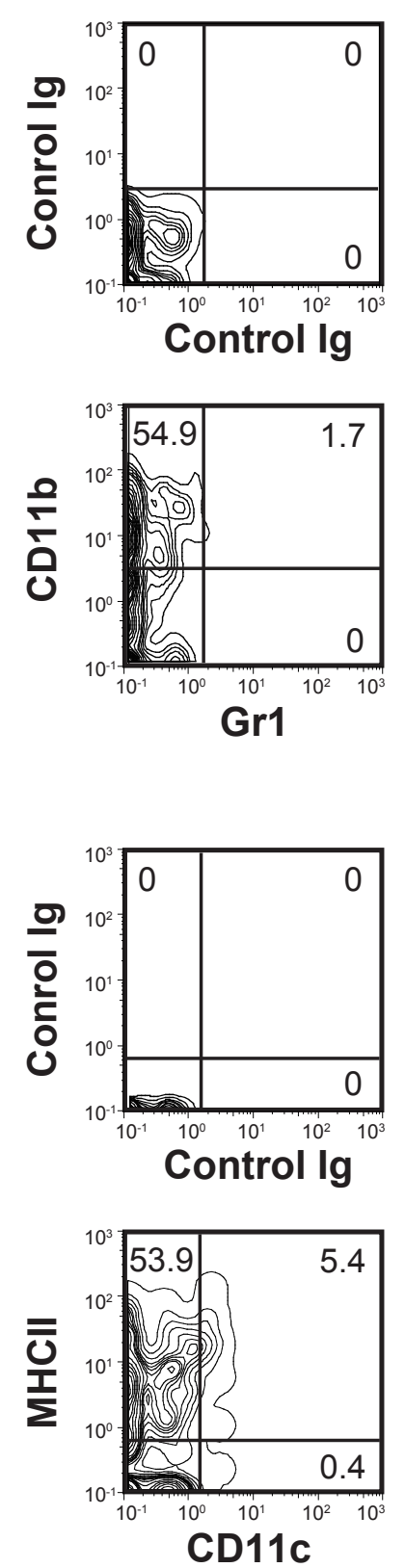
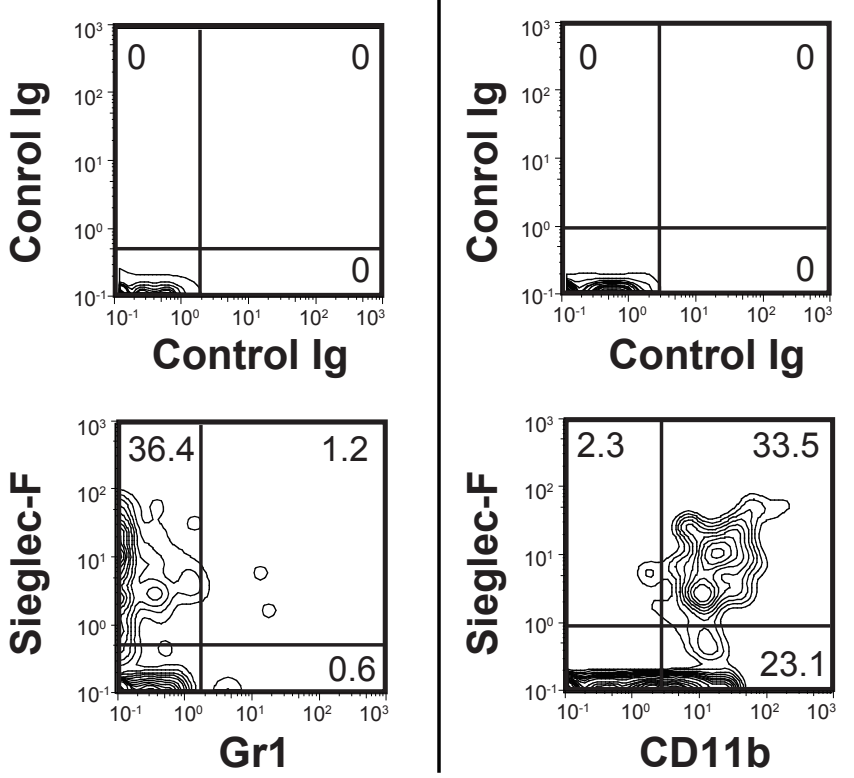
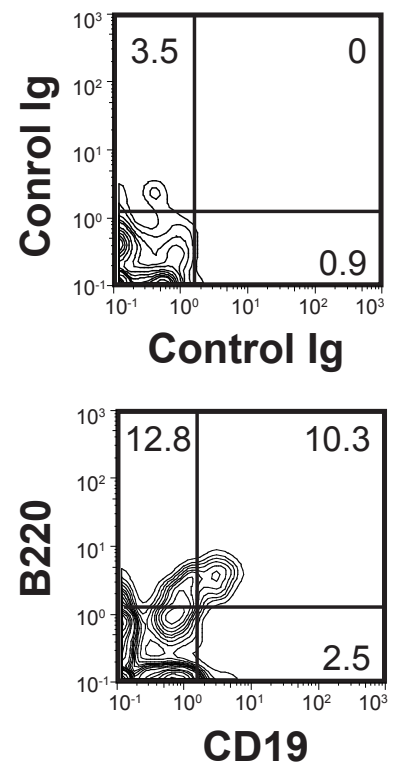
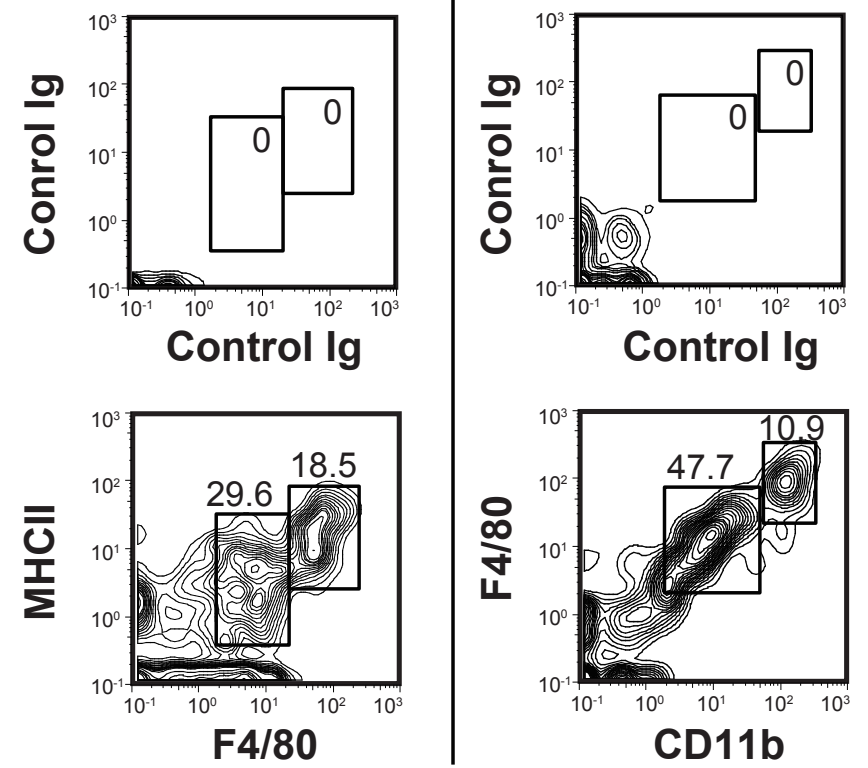
Figure 4. Shimura et al.

A

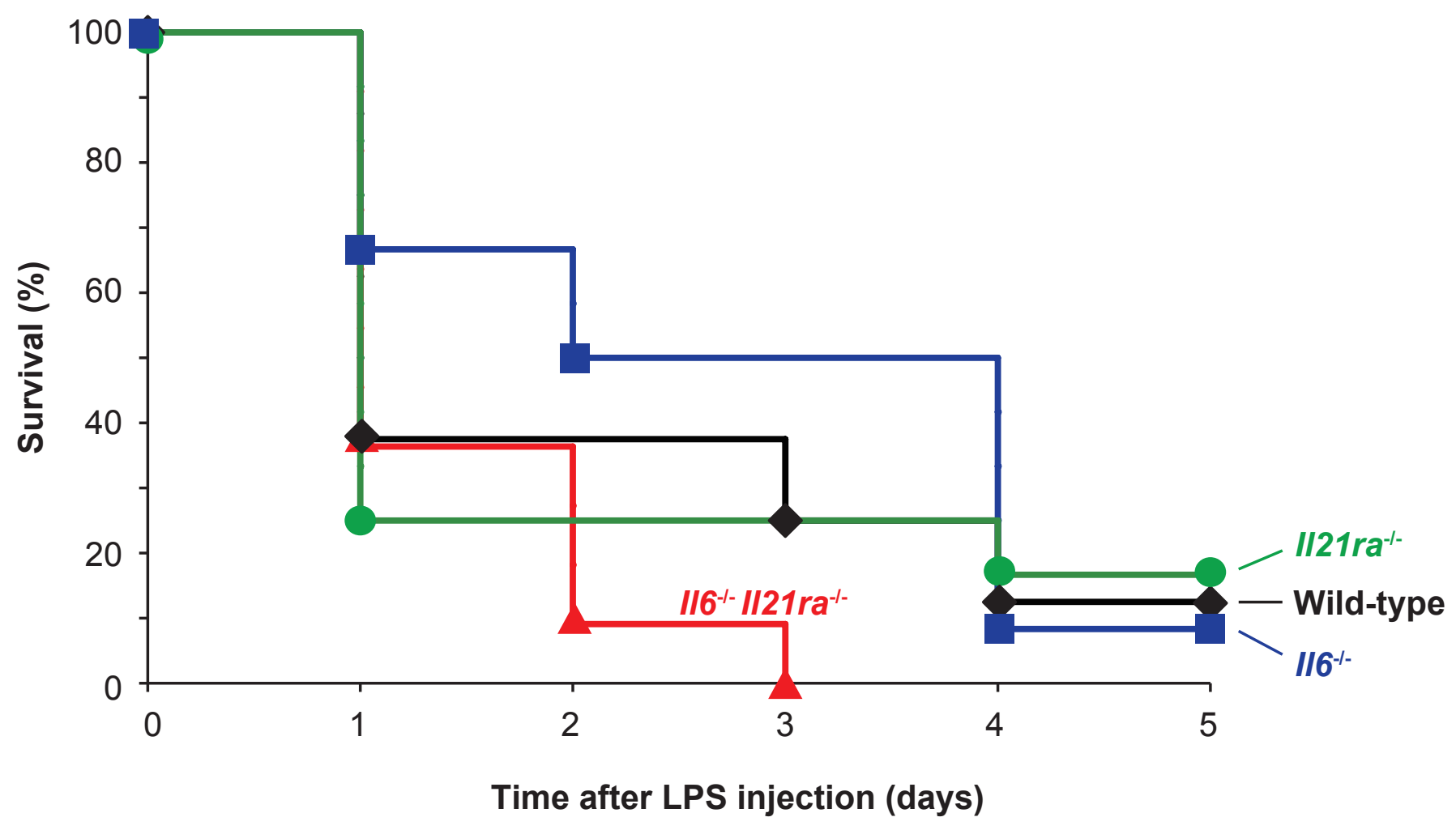

B

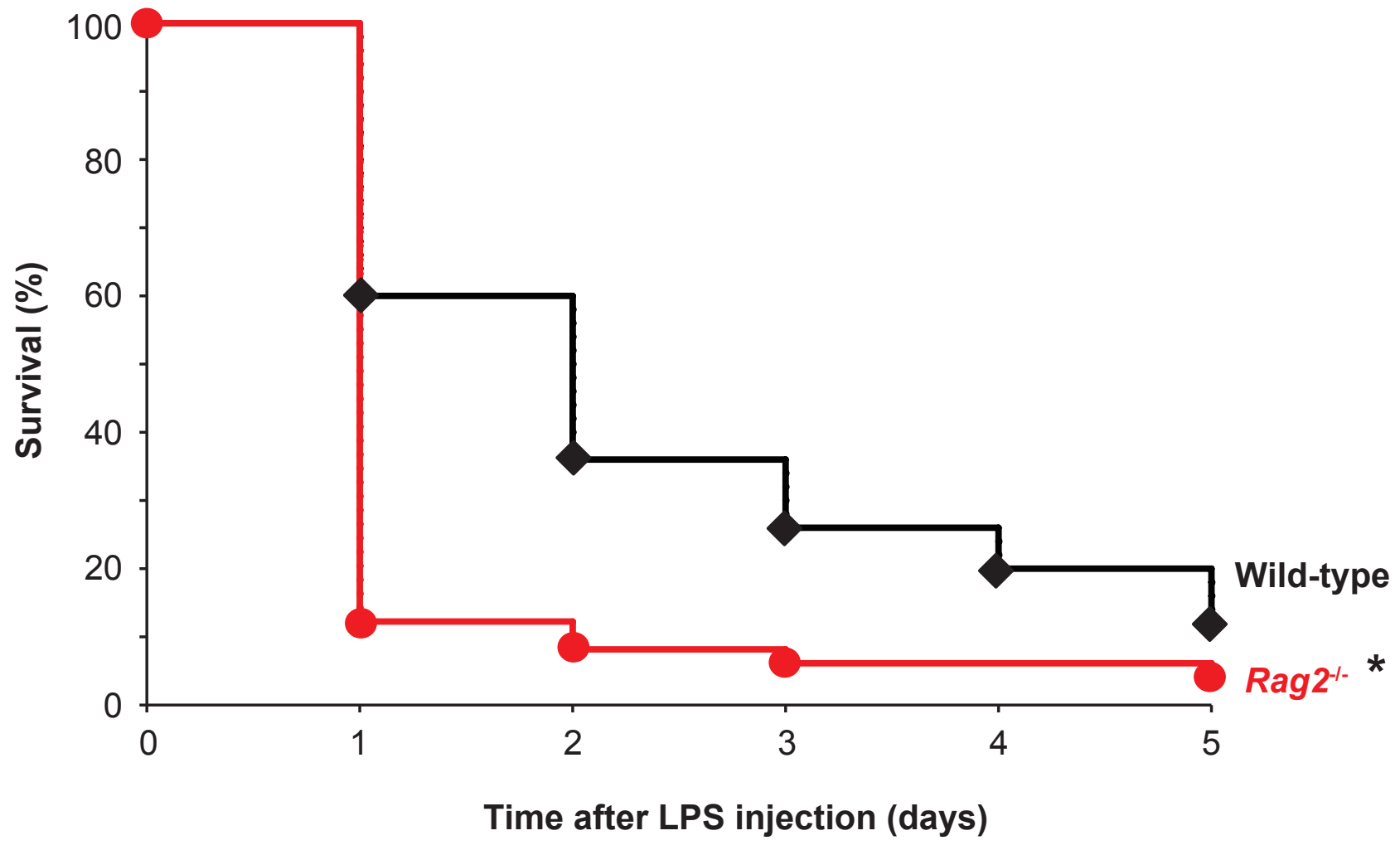

